CRYSTALLOGRAPHIC COMMUNICATIONS

ISSN 2056-9890

Received 11 June 2021

Accepted 22 June 2021

Edited by A. V. Yatsenko, Moscow State University, Russia

Keywords: crystal structure; 1,3-diazinane ring; hydrogen bonds; Hirshfeld surface analysis.

CCDC reference: 2091530

Supporting information: this article has supporting information at journals.iucr.org/e

\section{Crystal structure and Hirshfeld surface analysis of dimethyl 5-[2-(2,4,6-trioxo-1,3-diazinan-5-yl- idene)hydrazin-1-yl]benzene-1,3-dicarboxylate 0.224-hydrate}

Zeliha Atioğlu, ${ }^{a}$ Mehmet Akkurt, ${ }^{\text {b }}$ Gunay Z. Mammadova, ${ }^{\text {c }}$ Fatali E. Huseynov, ${ }^{\text {d }}$ Sevinj R. Hajiyeva, ${ }^{\mathrm{c}}$ Nazim T. Shamilov ${ }^{\mathrm{c}}$ and Ajaya Bhattarai ${ }^{\mathrm{e}}$

a Department of Aircraft Electrics and Electronics, School of Applied Sciences, Cappadocia University, Mustafapaşa, 50420 Ürgüp, Nevşehir, Turkey, bDepartment of Physics, Faculty of Sciences, Erciyes University, 38039 Kayseri, Turkey, ${ }^{\mathbf{c}}$ Department of Chemistry, Baku State University, Z. Khalilov str. 23, AZ, 1148 Baku, Azerbaijan, ${ }^{\mathbf{d} D e p a r t m e n t ~ o f ~ E c o l o g y ~}$ and Soil Sciences, Baku State University, Z. Khalilov str. 23, AZ, 1148 Baku, Azerbaijan, and ' Department of Chemistry, M.M.A.M.C (Tribhuvan University) Biratnagar, Nepal. *Correspondence e-mail: bkajaya@yahoo.com

In the crystal, the whole molecule of the title compound, $\mathrm{C}_{14} \mathrm{H}_{12} \mathrm{~N}_{4} \mathrm{O}_{7} \cdot 0.224 \mathrm{H}_{2} \mathrm{O}$, is nearly planar with a maximum deviation from the least-squares plane of 0.352 (1) $\AA$. The molecular conformation is stabilized by an intramolecular $\mathrm{N}-$ $\mathrm{H}$. . O hydrogen bond, generating an $S(6)$ ring motif. In the crystal, molecules are linked by centrosymmetric pairs of $\mathrm{N}-\mathrm{H} \cdots \mathrm{O}$ hydrogen bonds, forming ribbons along the $c$-axis direction. These ribbons connected by van der Waals contacts, forming sheets parallel to the $a c$ plane. There are also intermolecular van der Waals contacts and and $\mathrm{C}-\mathrm{H} \cdots \pi$ interactions between the sheets. A Hirshfeld surface analysis indicates that the most prevalent interactions are $\mathrm{O} \cdots \mathrm{H} / \mathrm{H} \cdots \mathrm{O}(41.2 \%), \mathrm{H} \cdots \mathrm{H}(19.2 \%), \mathrm{C} \cdots \mathrm{H} / \mathrm{H} \cdots \mathrm{C}(12.2 \%)$ and $\mathrm{C} \cdots \mathrm{O} /$ O. . C $(8.4 \%)$.

\section{Chemical context}

Arylhydrazones, besides their biological significance (Viswanathan et al., 2019), can also be used as precursors in the synthesis of coordination compounds (Gurbanov et al., 2017, 2018a,b; Ma et al., 2017a,b) and as building blocks in the construction of supramolecular structures owing to their hydrogen-bond donor and acceptor capabilities (Mahmoudi et al., 2016, 2017a,b,c, 2018a,b; 2019). All the reported hydrazone ligands are stabilized in the hydrazone form by intramolecular resonance-assisted hydrogen bonding (RAHB) between the hydrazone $=\mathrm{N}-\mathrm{NH}-$ fragment and the carbonyl group, giving a six-membered ring (Gurbanov et al., 2020a; Kopylovich et al., 2011a,b; Mizar et al., 2012). The use of multifunctional ligands in coordination chemistry is a common way to increase the water solubility of metal complexes, which is important for catalytic applications in aqueous medium (Ma et al., 2020, 2021; Mahmudov et al., 2013; Sutradhar et al., 2015, 2016). Moreover, non-covalent interactions such as hydrogen, halogen and chalcogen bonds as well as $\pi$-interactions or their cooperation are able to contribute to synthesis and catalysis and improve the properties of materials (Gurbanov et al., 2020b; Karmakar et al., 2017; Khalilov et al., 2018a,b; Mac Leod et al., 2012; Shikhaliyev et al., 2019; Shixaliyev et al., 2014). For that, the main skeleton of the hydrazone ligand should be decorated by non-covalent bond donor centre(s). In 
a continuation of our work in this area, we have prepared a new hydrazone ligand, dimethyl 5-\{2-[2,4,6-trioxotetrahydropyrimidin-5(2H)-ylidene] hydrazineyl\}isophthalate, which provides the centres for coordination and intermolecular noncovalent interactions.<smiles>COC(=O)c1cc(NN=C2C(=O)NC(=O)NC2=O)cc(C(=O)OC)c1</smiles>

\section{Structural commentary}

The asymmetric unit of the title structure contains one title molecule and a water molecule, which partially occupies a small cavity with an occupancy factor of 0.224 (5). The title molecule (Fig. 1) is nearly planar with the largest deviation from the least-squares plane being 0.352 (1) $\AA$ for the methylcarboxylate atom O6. The 1,3-diazinane ring makes a dihedral angle of $9.96(5)^{\circ}$ with the benzene ring. The planar molecular conformation is stabilized by an intramolecular $\mathrm{N}-$ $\mathrm{H}$.. O contact (Table 1), generating an $S(6)$ ring motif (Bernstein et al., 1995).

\section{Supramolecular features}

In the crystal, the molecules are linked by pairs of $\mathrm{N}-\mathrm{H} \cdots \mathrm{O}$ hydrogen bonds into ribbons along the $c$-axis direction (Table 1). These ribbons are connected by van der Waals interactions, forming sheets parallel to the $a c$ plane. There are

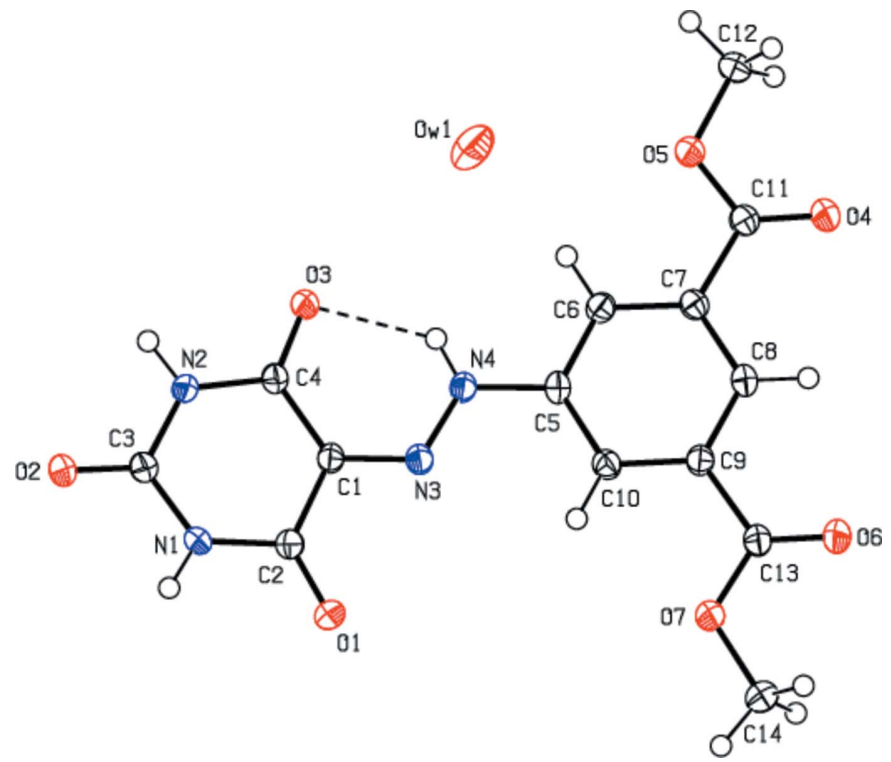

Figure 1

The molecular structure of the title compound, showing the atom labelling and displacement ellipsoids drawn at the $50 \%$ probability level.

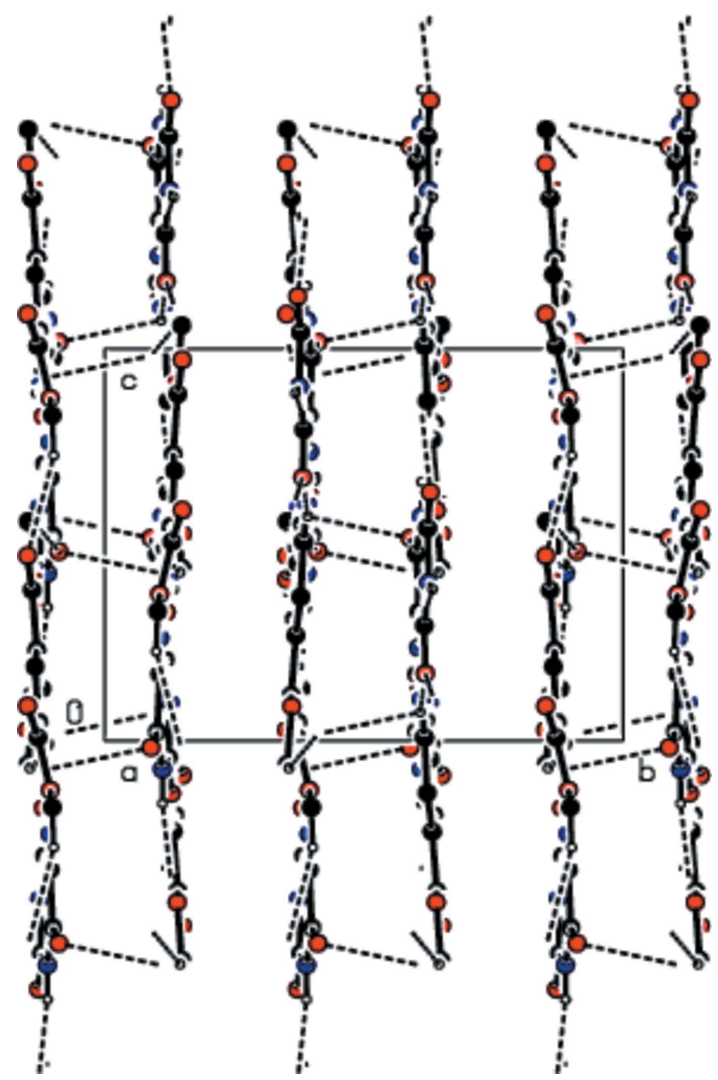

Figure 2

A view down the $a$ axis showing the intermolecular contacts forming the layered structure.

also other van der Waals contacts and $\mathrm{C}-\mathrm{H} \cdots \pi$ interactions between the sheets (Table 2), consolidating the crystal packing (Figs. 2-4).

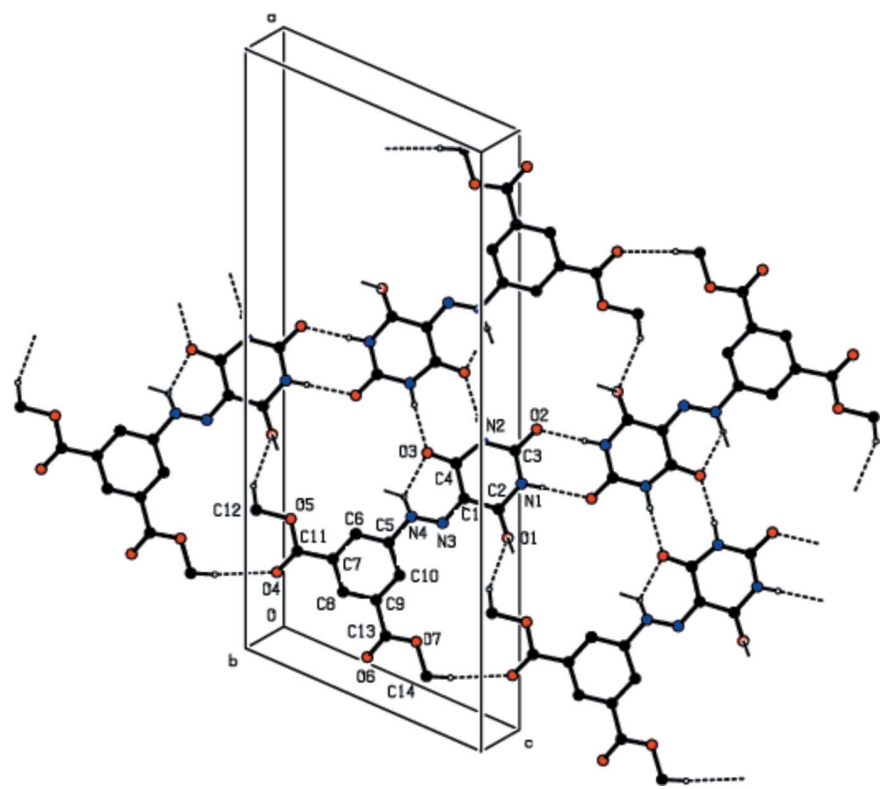

Figure 3

A view of intermolecular hydrogen bonds forming the ribbons along the $c$-axis direction. 
Table 1

Hydrogen-bond geometry $\left(\AA,^{\circ}\right)$.

$C g 2$ is the centroid of the $\mathrm{C} 5-\mathrm{C} 10$ benzene ring.

\begin{tabular}{lllll}
\hline$D-\mathrm{H} \cdots A$ & $D-\mathrm{H}$ & $\mathrm{H} \cdots A$ & $D \cdots A$ & $D-\mathrm{H} \cdots A$ \\
\hline $\mathrm{N} 1-\mathrm{H} 1 N \cdots \mathrm{O} 2^{\mathrm{i}}$ & 0.86 & 2.03 & $2.8800(13)$ & 174 \\
$\mathrm{~N} 2-\mathrm{H} 2 N \cdots \mathrm{O} 3^{\mathrm{ii}}$ & 0.90 & 2.01 & $2.8931(15)$ & 168 \\
$\mathrm{~N} 4-\mathrm{H} 4 N \cdots \mathrm{O} 3$ & 0.86 & 2.02 & $2.6571(15)$ & 131 \\
$\mathrm{C} 6-\mathrm{H} 6 \cdots \mathrm{O} 1$ & 0.95 & 2.14 & $3.061(6)$ & 163 \\
$\mathrm{C} 12-\mathrm{H} 12 B \cdots \mathrm{O} 1^{\text {iii }}$ & 0.98 & 2.39 & $3.2743(17)$ & 149 \\
$\mathrm{C}^{\mathrm{iv}}-\mathrm{H} 14 B \cdots 4^{\mathrm{v}}$ & 0.98 & 2.53 & $3.4754(16)$ & 163 \\
$\mathrm{C}^{2}-\mathrm{H} 12 C \cdots C g 2^{\mathrm{v}}$ & 0.98 & 2.73 & $3.4717(15)$ & 133 \\
\hline
\end{tabular}

Symmetry codes: (i) $-x+1, y,-z+\frac{5}{2}$; (ii) $-x+1, y,-z+\frac{3}{2}$; (iii) $x, y, z-1$; (iv) $x, y, z+1 ;$ (v) $x,-y+1, z-\frac{1}{2}$.

Table 2

Summary of short interatomic contacts ( $\mathrm{A})$ in the title compound.

\begin{tabular}{|c|c|c|}
\hline Contact & Distance & Symmetry operation \\
\hline $\mathrm{O} 1 \ldots * \mathrm{Ow} 1$ & 3.129 & $x, y, 1+z$ \\
\hline $\mathrm{O} 1 \cdots \mathrm{H} 12 B$ & 2.39 & $x, y, 1+z$ \\
\hline $\mathrm{O} 1 \cdots \mathrm{H} 4 N$ & 2.59 & $x, 1-y, \frac{1}{2}+z$ \\
\hline $\mathrm{H} 12 A \cdots \mathrm{O} 1$ & 2.67 & $\frac{1}{2}-x, \frac{1}{2}-y, 1-z$ \\
\hline $\mathrm{H} 1 N \cdots \mathrm{O} 2$ & 2.03 & $1-x, y, \frac{5}{2}-z$ \\
\hline $\mathrm{O} 2 \ldots * \mathrm{Ow} 1$ & 2.662 & $1-x, y, \frac{3}{2}-z$ \\
\hline $\mathrm{N} 2 \ldots \mathrm{O} 2$ & 3.226 & $1-x, 1-y, 2-z$ \\
\hline $\mathrm{O} 2 \cdots \mathrm{H} 14 C$ & 2.64 & $\frac{1}{2}+x, \frac{1}{2}-y, \frac{1}{2}+z$ \\
\hline $\mathrm{H} 2 N \cdots \mathrm{O} 3$ & 2.01 & $1-x, y, \frac{3}{2}-z$ \\
\hline $\mathrm{H} 4 N \cdots \mathrm{O} 1$ & 2.59 & $x, 1-y,-\frac{1}{2}+z$ \\
\hline $\mathrm{H} 12 B \cdots \mathrm{O} 1$ & 2.39 & $x, y,-1+z$ \\
\hline 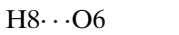 & 2.66 & $-x, y, \frac{1}{2}-z$ \\
\hline $\mathrm{H} 14 A \cdots \mathrm{O} 6$ & 2.67 & $-x, 1-y, 1-z$ \\
\hline $\mathrm{H} 14 C \cdots \mathrm{O} 2$ & 2.64 & $-\frac{1}{2}+x, \frac{1}{2}-y,-\frac{1}{2}+z$ \\
\hline $\mathrm{C} 1 \ldots * \mathrm{O} w 1$ & 3.297 & $x, 1-y, \frac{1}{2}+z$ \\
\hline H6 . **ow1 & 2.14 & $x, y, z$ \\
\hline $\mathrm{H} 12 B \cdots \mathrm{C} 12$ & 3.10 & $\frac{1}{2}-x, \frac{1}{2}-y,-z$ \\
\hline $\mathrm{H} 14 B \cdots \mathrm{C} 14$ & 2.93 & $-x, y, \frac{3}{2}-z$ \\
\hline $\mathrm{H} 12 A \ldots * \mathrm{Ow} 1$ & 2.70 & $\frac{1}{2}-x, \frac{1}{2}-y,-z$ \\
\hline
\end{tabular}

*Ow1 indicates the oxygen atom of the water molecule with an occupancy of 0.224 (5).

\section{Hirshfeld surface analysis}

The Hirshfeld surface for the title molecule was performed and its associated two-dimensional fingerprint plots were prepared using Crystal Explorer 17 (Turner et al., 2017) to further investigate the intermolecular interactions in the title structure. The oxygen atom of the water molecule with partial occupancy was not taken into account. The Hirshfeld surface mapped over $d_{\text {norm }}$ with corresponding colours representing intermolecular interactions is shown in Fig. 5. The red spots on the surface correspond to the $\mathrm{N}-\mathrm{H} \cdots \mathrm{O}$ and $\mathrm{C}-\mathrm{H} \cdots \mathrm{O}$

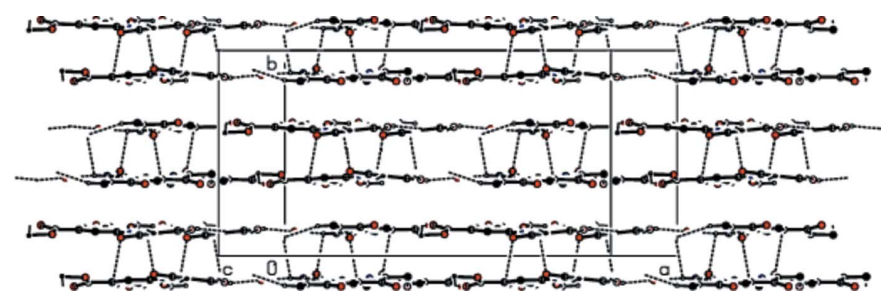

Figure 4

A view of the projection on the $a b$ plane showing the contacts between layers.

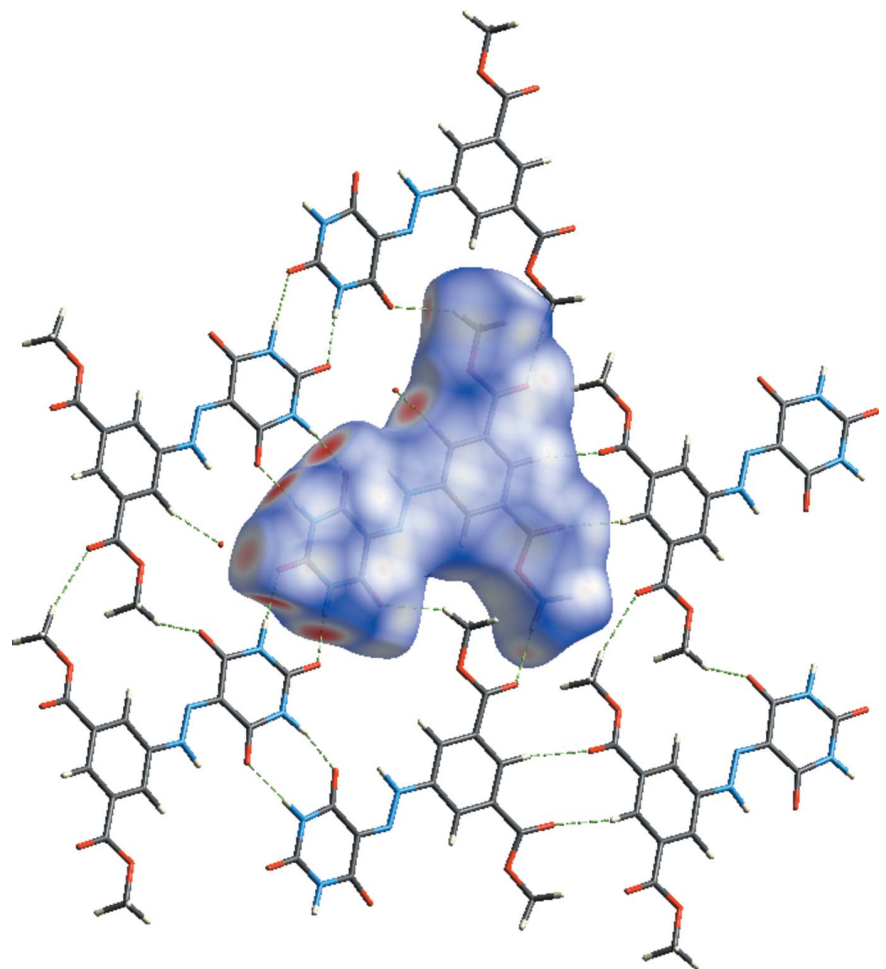

Figure 5

A view of the Hirshfeld surface mapped over $d_{\text {norm }}$, with interactions to neighbouring molecules shown as green dashed lines.

interactions (Tables 1 and 2). The Hirshfeld surface mapped over electrostatic potential (Spackman et al., 2009) is shown in Fig. 6 . The blue regions indicate positive electrostatic potential

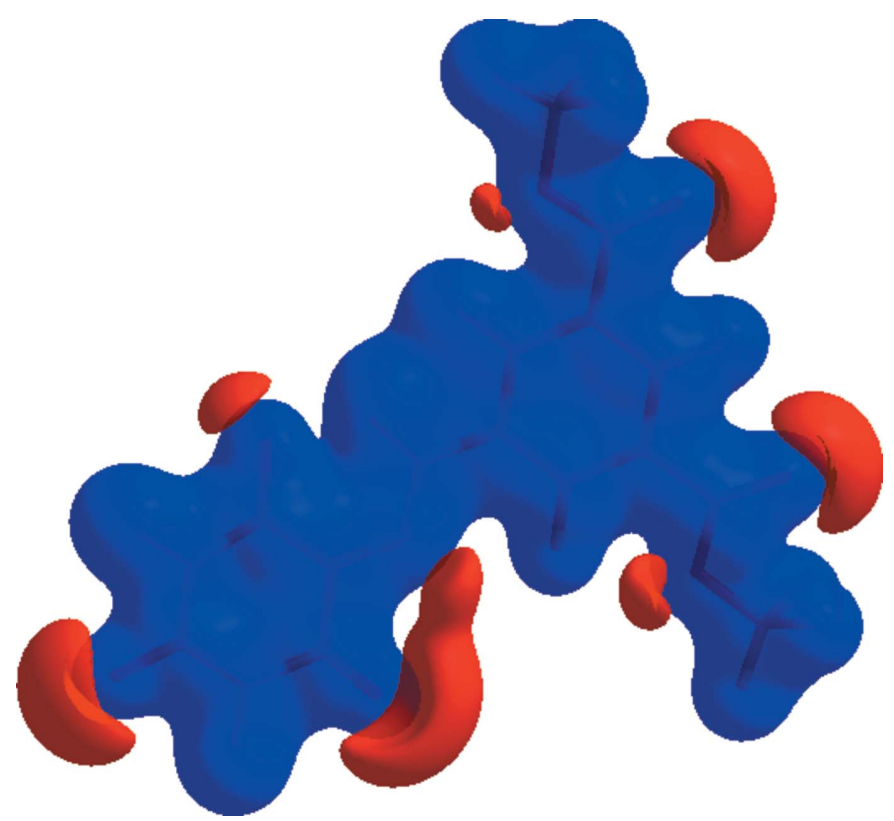

Figure 6

The Hirshfeld surface of the title compound plotted over electrostatic potential energy in the range from -0.0500 to 0.0500 a.u. using the STO$3 \mathrm{G}$ basis set at the Hartree-Fock level of theory. Hydrogen-bond donors and acceptors are shown as blue and red regions around the atoms, corresponding to positive and negative potentials, respectively. 


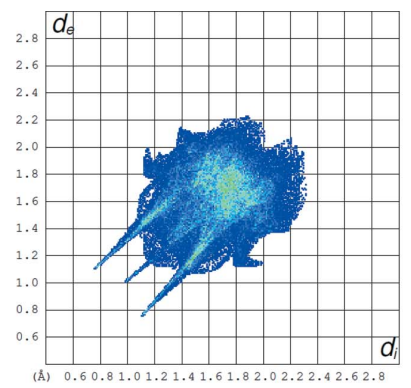

(a) All...All

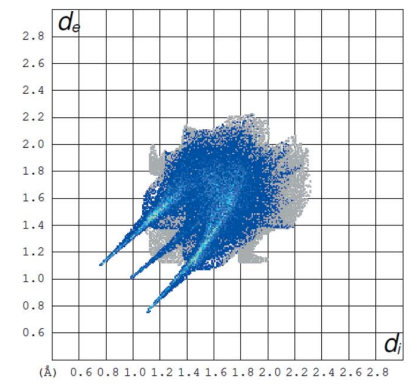

(b) $\mathbf{O} \cdots \mathbf{H} / \mathbf{H} \cdots \mathbf{O}$

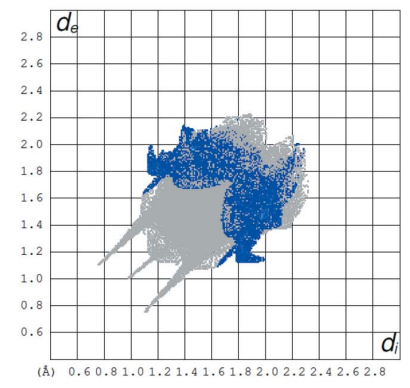

(d) $\mathbf{C} \cdots \mathbf{H} / \mathbf{H} \cdots \mathrm{C}$

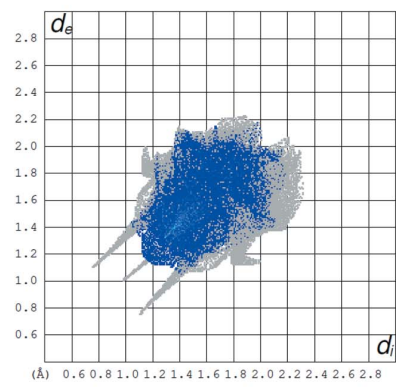

(c) $\mathbf{H} \cdots \mathbf{H}$

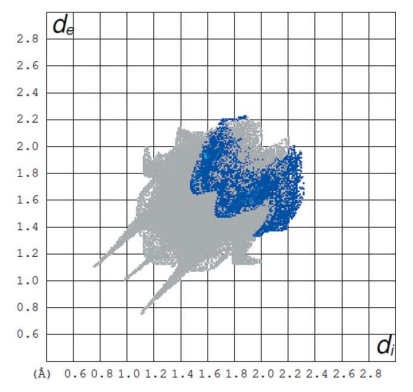

(e) $\mathrm{C} \cdots \mathbf{O} / \mathrm{O} \cdots \mathrm{C}$
Figure 7

The two-dimensional fingerprint plots of the title compound, showing $(a)$ all interactions, and delineated into $(b) \mathrm{O} \cdots \mathrm{H} / \mathrm{H} \cdots \mathrm{O},(c) \mathrm{H} \cdots \mathrm{H},(d)$ $\mathrm{C} \cdots \mathrm{H} / \mathrm{H} \cdots \mathrm{C}$ and $(e) \mathrm{C} \cdots \mathrm{O} / \mathrm{O} \cdots \mathrm{C}$, interactions $\left[d_{\mathrm{e}}\right.$ and $d_{\mathrm{i}}$ represent the distances from a point on the Hirshfeld surface to the nearest atoms outside (external) and inside (internal) the surface, respectively].

(hydrogen-bond donors), while the red regions indicate negative electrostatic potential (hydrogen-bond acceptors). The two-dimensional fingerprint plots (McKinnon et al., 2007) are shown in Fig. 7. O $\cdots \mathrm{H} / \mathrm{H} . \mathrm{O}$ contacts make the largest contribution ( $41.2 \%$; Fig. $7 b$ ) to the Hirshfeld surface. The other large contributions to the Hirshfeld surface are from $\mathrm{H} \cdots \mathrm{H}(19.2 \%$; Fig. 7c), C. $\mathrm{H} / \mathrm{H} \cdots \mathrm{C}(12.2 \%$; Fig. $7 d)$ and $\mathrm{C} \cdots \mathrm{O} / \mathrm{O} \cdots \mathrm{C}(8.4 \%$; Fig. $7 e)$ interactions. All contributions to the Hirshfeld surface are listed in Table 3. These interactions play a crucial role in the overall cohesion of the crystal packing.

\section{Database survey}

A search of Cambridge Crystallographic Database (CSD, version 5.40, update of September 2019; Groom et al., 2016)
Table 3

Percentage contributions of interatomic contacts to the Hirshfeld surface for the title compound.

\begin{tabular}{ll}
\hline Contact & Percentage contribution \\
\hline $\mathrm{O} \cdots \mathrm{H} / \mathrm{H} \cdots \mathrm{O}$ & 41.2 \\
$\mathrm{H} \cdots \mathrm{H}$ & 19.2 \\
$\mathrm{C} \cdots \mathrm{H} / \mathrm{H} \cdots \mathrm{C}$ & 12.2 \\
$\mathrm{C} \cdots \mathrm{O} / \mathrm{O} \cdots \mathrm{C}$ & 8.4 \\
$\mathrm{O} \cdots \mathrm{O}$ & 5.6 \\
$\mathrm{~N} \cdots \mathrm{O} / \mathrm{O} \cdots \mathrm{N}$ & 4.7 \\
$\mathrm{C} \cdots \mathrm{N} / \mathrm{N} \cdots \mathrm{C}$ & 3.2 \\
$\mathrm{C} \cdots \mathrm{C}$ & 2.8 \\
$\mathrm{~N} \cdots \mathrm{H} / \mathrm{H} \cdots \mathrm{N}$ & 2.7 \\
\hline
\end{tabular}

was undertaken for structures containing the 5-(2-methylhydrazinylidene)-1,3-diazinane moiety. The first three structures are free bases are: 2-\{2-[(1H-imidazol-5-yl)methylidene]1-methylhydrazinyl pyridine (QUGVEW; Bocian et al., 2020), 2-\{2-[(1H-imidazol-2-yl)methylidene]-1-methylhydrazinyl\}$1 H$-benzimidazole monohydrate (QUGVIA; Bocian et al., 2020) and 2-\{1-methyl-2-[(1-methyl- $1 H$-imidazol-2-yl)methylidene]hydrazinyl $\}-1 H$-benzimidazole hydrate unknown solvate (QUGVOG; Bocian et al., 2020). The other two are triflate salts are: 5-\{[2-(1H-benzimidazol-2-yl)-2-methylhydrazinylidene]methyl $\}-1 H$-imidazol-3-ium trifluoromethanesulfonate monohydrate (QUGVUM; Bocian et al., 2020) and $\quad(2-\{2-[(1 H$-imidazol-3-ium-2-yl)methylene $]-1-m e t h y l-$ hydrazineyl\}pyridin-1-ium) bis(trifluoromethanesulfonate) (QUGWAT; Bocian et al., 2020).

In the structures of QUGVEW, QUGVIA, QUGVOG, QUGVUM and QUGWAT, the most important contribution to the stabilization of the crystal packing is provided by $\pi-\pi$ interactions, especially between cations in the structures of salts, while the characteristics of the crystal architecture are influenced by directional interactions, especially relatively strong hydrogen bonds. In one of the structures (QUGWAT), an interesting example of a non-typical $\mathrm{F}$... O interaction was found whose length, 2.859 (2) $\AA$, is one of the shortest ever reported.

\section{Synthesis and crystallization}

Diazotization: $2.09 \mathrm{~g} \quad(10 \mathrm{mmol})$ of dimethyl 5-aminoisophthalate were dissolved in $50 \mathrm{~mL}$ of water, the solution was cooled on an ice bath to $273 \mathrm{~K}$ and $0.69 \mathrm{~g}(10 \mathrm{mmol})$ of $\mathrm{NaNO}_{2}$ were added; $2.00 \mathrm{~mL}$ of $\mathrm{HCl}$ were then added in $0.5 \mathrm{~mL}$ portions over $1 \mathrm{~h}$. The temperature of the mixture should not exceed $278 \mathrm{~K}$.

Azocoupling: $\mathrm{NaOH}(0.40 \mathrm{~g}, 10 \mathrm{mmol})$ was added to a mixture of $10 \mathrm{mmol}(1.28 \mathrm{~g})$ of barbituric acid with $25.00 \mathrm{~mL}$ of water. The solution was cooled on an ice bath and a suspension of 3,5-bis(methoxycarbonyl)benzenediazonium chloride, prepared according to the procedure described above, was added in two equal portions under vigorous stirring for $1 \mathrm{~h}$. The formed precipitate of the title compound was filtered off, recrystallized from methanol and dried in air. 
Table 4

Experimental details.

\begin{tabular}{|c|c|}
\hline Crystal data & \\
\hline Chemical formula & $\mathrm{C}_{14} \mathrm{H}_{12} \mathrm{~N}_{4} \mathrm{O}_{7} \cdot 0.224 \mathrm{H}_{2} \mathrm{O}$ \\
\hline$M_{\mathrm{r}}$ & 351.86 \\
\hline Crystal system, space group & Monoclinic, $C 2 / c$ \\
\hline Temperature $(\mathrm{K})$ & 150 \\
\hline$a, b, c(\AA)$ & $\begin{array}{l}24.2097(11), 12.6311(6), \\
10.4022(5)\end{array}$ \\
\hline$\beta\left(^{\circ}\right)$ & $113.133(2)$ \\
\hline$V\left(\AA^{3}\right)$ & $2925.2(2)$ \\
\hline$Z$ & 8 \\
\hline Radiation type & Мo $K \alpha$ \\
\hline$\mu\left(\mathrm{mm}^{-1}\right)$ & 0.13 \\
\hline Crystal size $(\mathrm{mm})$ & $0.34 \times 0.32 \times 0.27$ \\
\hline Data collection & \\
\hline Diffractometer & Bruker APEXII CCD \\
\hline $\begin{array}{l}\text { No. of measured, independent and } \\
\text { observed }[I>2 \sigma(I)] \text { reflections }\end{array}$ & $34832,2944,2699$ \\
\hline$R_{\text {int }}$ & 0.017 \\
\hline$(\sin \theta / \lambda)_{\max }\left(\AA^{-1}\right)$ & 0.625 \\
\hline Refinement & \\
\hline$R\left[F^{2}>2 \sigma\left(F^{2}\right)\right], w R\left(F^{2}\right), S$ & $0.034,0.102,1.04$ \\
\hline No. of reflections & 2944 \\
\hline No. of parameters & 241 \\
\hline No. of restraints & 6 \\
\hline $\mathrm{H}$-atom treatment & $\begin{array}{l}\mathrm{H} \text { atoms treated by a mixture of } \\
\text { independent and constrained } \\
\text { refinement }\end{array}$ \\
\hline$\Delta \rho_{\max }, \Delta \rho_{\min }\left(\mathrm{e} \AA^{-3}\right)$ & $0.29,-0.21$ \\
\hline
\end{tabular}

Computer programs: APEX2 and SAINT (Bruker, 2007), SHELXT (Sheldrick, 2015a), SHELXL (Sheldrick, 2015b), ORTEP-3 for Windows (Farrugia, 2012) and PLATON (Spek, 2020).

Crystals suitable for X-ray analysis were obtained by slow evaporation of an ethanol solution.

The title compound: Yield, $68 \%$ (based on barbituric acid), yellow powder soluble in DMSO, methanol, ethanol and DMF. Analysis calculated for $\mathrm{C}_{14} \mathrm{H}_{12} \mathrm{~N}_{4} \mathrm{O}_{7}\left(M_{\mathrm{r}}=348.27\right)$ : C, 48.28; $\mathrm{H}$, 3.47; N, 16.09; found: C, $48.25 \mathrm{H}, 3.41$; N, 16.03\%. ESI-MS: $m /$ $z: 349.2\left[M_{\mathrm{r}}+\mathrm{H}\right]^{+}$. IR (KBr): 3160, 3090 and $2846 v(\mathrm{NH}), 1745$ and $1663 v(\mathrm{C}=\mathrm{O}), 1610 v(\mathrm{C}=\mathrm{O} \cdots \mathrm{H}) \mathrm{cm}^{-1} \cdot{ }^{1} \mathrm{H}$ NMR (300.130 MHz) in DMSO- $d_{6}$, internal TMS, $\delta(\mathrm{ppm}): 8.20-8.36$ $(3 \mathrm{H}, \mathrm{Ar}-\mathrm{H}), 11.32(s, 1 \mathrm{H}, \mathrm{N}-\mathrm{H}), 11.54(s, 1 \mathrm{H}, \mathrm{N}-\mathrm{H}), 14.08$ $(s, 1 \mathrm{H}, \mathrm{N}-\mathrm{H}) .{ }^{13} \mathrm{C}\left\{{ }^{1} \mathrm{H}\right\} \mathrm{NMR}\left(75.468 \mathrm{MHz}, \mathrm{DMSO}-d_{6}\right) . \delta: 55.6$ $\left(2 \mathrm{OCH}_{3}\right), 119.54(2 \mathrm{Ar}-\mathrm{H}), 121.8(\mathrm{Ar}-\mathrm{H}), 127.4(2 \mathrm{C}-$ $\left.\mathrm{COOCH}_{3}\right), 133.25(\mathrm{C}=\mathrm{N}), 142.87(\mathrm{C}-\mathrm{NHN}=), 150.24$ $(\mathrm{C}=\mathrm{O}), 160.32(\mathrm{C}=\mathrm{O}), 161.90(\mathrm{C}=\mathrm{O} \cdots \mathrm{H})$ and 166.56 $\left(2 \mathrm{COOCH}_{3}\right)$.

\section{Refinement details}

Crystal data, data collection and structure refinement details are summarized in Table 4. The $\mathrm{H}$ atoms of the $\mathrm{NH}$ groups were located by difference Fourier synthesis and their coordinates were fixed. All C-bound $\mathrm{H}$ atoms were positioned geometrically and refined using a riding model, with $\mathrm{C}-\mathrm{H}=$ 0.95 and $0.98 \AA$, and with $U_{\text {iso }}(\mathrm{H})=1.2$ or $1.5 U_{\text {eq }}(\mathrm{C})$. There is a small cavity in the crystal, which is only partially occupied by a water molecule, with an occupancy of 0.224 (5), and its hydrogen atoms could not be located.

\section{Acknowledgements}

The authors' contributions are as follows. Conceptualization, ZA, MA, GZM, FEH, SRH, NTS, and AB; methodology, SRH, and NTS; investigation, ZA, and GZM; writing (original draft), FEH, MA and AB; writing (review and editing of the manuscript), $\mathrm{MA}$ and $\mathrm{AB}$; crystal-structure determination, GZM; visualization, ZA, and MA; funding acquisition, GZM, FEH, SRH, and NTS; resources, ZA, MA and AB; supervision, $\mathrm{MA}$ and $\mathrm{AB}$.

\section{Funding information}

This work was supported by Baku State University.

\section{References}

Bernstein, J., Davis, R. E., Shimoni, L. \& Chang, N.-L. (1995). Angew. Chem. Int. Ed. Engl. 34, 1555-1573.

Bocian, A., Gorczyński, A., Marcinkowski, D., Dutkiewicz, G., Patroniak, V. \& Kubicki, M. (2020). Acta Cryst. C76, 367-374.

Bruker (2007). APEX2 and SAINT. Bruker AXS Inc., Madison, Wisconsin, USA.

Farrugia, L. J. (2012). J. Appl. Cryst. 45, 849-854.

Groom, C. R., Bruno, I. J., Lightfoot, M. P. \& Ward, S. C. (2016). Acta Cryst. B72, 171-179.

Gurbanov, A. V., Kuznetsov, M. L., Demukhamedova, S. D., Alieva, I. N., Godjaev, N. M., Zubkov, F. I., Mahmudov, K. T. \& Pombeiro, A. J. L. (2020a). CrystEngComm, 22, 628-633.

Gurbanov, A. V., Kuznetsov, M. L., Mahmudov, K. T., Pombeiro, A. J. L. \& Resnati, G. (2020b). Chem. Eur. J. 26, 14833-14837.

Gurbanov, A. V., Maharramov, A. M., Zubkov, F. I., Saifutdinov, A. M. \& Guseinov, F. I. (2018a). Aust. J. Chem. 71, 190-194.

Gurbanov, A. V., Mahmoudi, G., Guedes da Silva, M. F. C., Zubkov, F. I., Mahmudov, K. T. \& Pombeiro, A. J. L. (2018b). Inorg. Chim. Acta, 471, 130-136.

Gurbanov, A. V., Mahmudov, K. T., Sutradhar, M., Guedes da Silva, F. C., Mahmudov, T. A., Guseinov, F. I., Zubkov, F. I., Maharramov, A. M. \& Pombeiro, A. J. L. (2017). J. Organomet. Chem. 834, 22-27. Karmakar, A., Rúbio, G. M. D. M., Paul, A., Guedes da Silva, M. F. C., Mahmudov, K. T., Guseinov, F. I., Carabineiro, S. A. C. \& Pombeiro, A. J. L. (2017). Dalton Trans. 46, 8649-8657.

Khalilov, A. N., Asgarova, A. R., Gurbanov, A. V., Maharramov, A. M., Nagiyev, F. N. \& Brito, I. (2018a). Z. Kristallogr. New Cryst. Struct. 233, 1019-1020.

Khalilov, A. N., Asgarova, A. R., Gurbanov, A. V., Nagiyev, F. N. \& Brito, I. (2018b). Z. Kristallogr. New Cryst. Struct. 233, 947-948.

Kopylovich, M. N., Mahmudov, K. T., Haukka, M., Luzyanin, K. V. \& Pombeiro, A. J. L. (2011a). Inorg. Chim. Acta, 374, 175-180.

Kopylovich, M. N., Mahmudov, K. T., Mizar, A. \& Pombeiro, A. J. L. (2011b). Chem. Commun. 47, 7248-7250.

Ma, Z., Gurbanov, A. V., Maharramov, A. M., Guseinov, F. I., Kopylovich, M. N., Zubkov, F. I., Mahmudov, K. T. \& Pombeiro, A. J. L. (2017a). J. Mol. Catal. A Chem. 426, 526-533.

Ma, Z., Gurbanov, A. V., Sutradhar, M., Kopylovich, M. N., Mahmudov, K. T., Maharramov, A. M., Guseinov, F. I., Zubkov, F. I. \& Pombeiro, A. J. L. (2017b). Mol. Catal. 428, 17-23.

Ma, Z., Mahmudov, K. T., Aliyeva, V. A., Gurbanov, A. V., Guedes da Silva, M. F. C. \& Pombeiro, A. J. L. (2021). Coord. Chem. Rev. 437, 213859.

Ma, Z., Mahmudov, K. T., Aliyeva, V. A., Gurbanov, A. V. \& Pombeiro, A. J. L. (2020). Coord. Chem. Rev. 423, 213482.

Mac Leod, T. C. O., Kopylovich, M. N., Guedes da Silva, M. F. C., Mahmudov, K. T. \& Pombeiro, A. J. L. (2012). Appl. Catal. Gen. 439-440, 15-23. 
Mahmoudi, G., Bauzá, A., Gurbanov, A. V., Zubkov, F. I., Maniukiewicz, W., Rodríguez-Diéguez, A., López-Torres, E. \& Frontera, A. (2016). CrystEngComm, 18, 9056-9066.

Mahmoudi, G., Dey, L., Chowdhury, H., Bauzá, A., Ghosh, B. K., Kirillov, A. M., Seth, S. K., Gurbanov, A. V. \& Frontera, A. (2017a). Inorg. Chim. Acta, 461, 192-205.

Mahmoudi, G., Gurbanov, A. V., Rodríguez-Hermida, S., Carballo, R., Amini, M., Bacchi, A., Mitoraj, M. P., Sagan, F., Kukułka, M. \& Safin, D. A. (2017b). Inorg. Chem. 56, 9698-9709.

Mahmoudi, G., Khandar, A. A., Afkhami, F. A., Miroslaw, B., Gurbanov, A. V., Zubkov, F. I., Kennedy, A., Franconetti, A. \& Frontera, A. (2019). CrystEngComm, 21, 108-117.

Mahmoudi, G., Seth, S. K., Bauzá, A., Zubkov, F. I., Gurbanov, A. V., White, J., Stilinović, V., Doert, T. \& Frontera, A. (2018a). CrystEngComm, 20, 2812-2821.

Mahmoudi, G., Zangrando, E., Mitoraj, M. P., Gurbanov, A. V., Zubkov, F. I., Moosavifar, M., Konyaeva, I. A., Kirillov, A. M. \& Safin, D. A. (2018b). New J. Chem. 42, 4959-4971.

Mahmoudi, G., Zaręba, J. K., Gurbanov, A. V., Bauzá, A., Zubkov, F. I., Kubicki, M., Stilinović, V., Kinzhybalo, V. \& Frontera, A. (2017c). Eur. J. Inorg. Chem. pp. 4763-4772.

Mahmudov, K. T., Kopylovich, M. N., Haukka, M., Mahmudova, G. S., Esmaeila, E. F., Chyragov, F. M. \& Pombeiro, A. J. L. (2013). J. Mol. Struct. 1048, 108-112.

McKinnon, J. J., Jayatilaka, D. \& Spackman, M. A. (2007). Chem. Commun. pp. 3814-3816.
Mizar, A., Guedes da Silva, M. F. C., Kopylovich, M. N., Mukherjee, S., Mahmudov, K. T. \& Pombeiro, A. J. L. (2012). Eur. J. Inorg. Chem. pp. 2305-2313.

Sheldrick, G. M. (2015a). Acta Cryst. A71, 3-8.

Sheldrick, G. M. (2015b). Acta Cryst. C71, 3-8.

Shikhaliyev, N. Q., Kuznetsov, M. L., Maharramov, A. M., Gurbanov, A. V., Ahmadova, N. E., Nenajdenko, V. G., Mahmudov, K. T. \& Pombeiro, A. J. L. (2019). CrystEngComm, 21, 5032-5038.

Shixaliyev, N. Q., Gurbanov, A. V., Maharramov, A. M., Mahmudov, K. T., Kopylovich, M. N., Martins, L. M. D. R. S., Muzalevskiy, V. M., Nenajdenko, V. G. \& Pombeiro, A. J. L. (2014). New J. Chem. 38, 4807-4815.

Spackman, M. A. \& Jayatilaka, D. (2009). CrystEngComm, 11, 19-32. Spek, A. L. (2020). Acta Cryst. E76, 1-11.

Sutradhar, M., Alegria, E. C. B. A., Mahmudov, K. T., Guedes da Silva, M. F. C. \& Pombeiro, A. J. L. (2016). RSC Adv. 6, 8079-8088. Sutradhar, M., Martins, L. M. D. R. S., Guedes da Silva, M. F. C., Mahmudov, K. T., Liu, C.-M. \& Pombeiro, A. J. L. (2015). Eur. J. Inorg. Chem. pp. 3959-3969.

Turner, M. J., McKinnon, J. J., Wolff, S. K., Grimwood, D. J., Spackman, P. R., Jayatilaka, D. \& Spackman, M. A. (2017). Crystal Explorer 17. The University of Western Australia.

Viswanathan, A., Kute, D., Musa, A., Mani, S. K., Sipilä, V., EmmertStreib, F., Zubkov, F. I., Gurbanov, A. V., Yli-Harja, O. \& Kandhavelu, M. (2019). Eur. J. Med. Chem. 166, 291-303. 


\section{supporting information}

Acta Cryst. (2021). E77, 759-764 [https://doi.org/10.1107/S2056989021006563]

Crystal structure and Hirshfeld surface analysis of dimethyl 5-[2-(2,4,6trioxo-1,3-diazinan-5-ylidene)hydrazin-1-yl]benzene-1,3-dicarboxylate 0.224hydrate

Zeliha Atioğlu, Mehmet Akkurt, Gunay Z. Mammadova, Fatali E. Huseynov, Sevinj R.

Hajiyeva, Nazim T. Shamilov and Ajaya Bhattarai

Computing details

Data collection: APEX2 (Bruker, 2007); cell refinement: SAINT (Bruker, 2007); data reduction: SAINT (Bruker, 2007); program(s) used to solve structure: SHELXT (Sheldrick, 2015a); program(s) used to refine structure: SHELXL

(Sheldrick, 2015b); molecular graphics: ORTEP-3 for Windows (Farrugia, 2012); software used to prepare material for publication: PLATON (Spek, 2020).

Dimethyl 5-[2-(2,4,6-trioxo-1,3-diazinan-5-ylidene)hydrazin-1-yl]benzene-1,3-dicarboxylate 0.224-hydrate

Crystal data

$\mathrm{C}_{14} \mathrm{H}_{12} \mathrm{~N}_{4} \mathrm{O}_{7} \cdot 0.224 \mathrm{H}_{2} \mathrm{O}$

$M_{r}=351.86$

Monoclinic, $C 2 / c$

$a=24.2097(11) \AA$

$b=12.6311(6) \AA$

$c=10.4022(5) \AA$

$\beta=113.133(2)^{\circ}$

$V=2925.2(2) \AA^{3}$

$Z=8$

$F(000)=1454$

$D_{\mathrm{x}}=1.598 \mathrm{Mg} \mathrm{m}^{-3}$

Mo $K \alpha$ radiation, $\lambda=0.71073 \AA$

Cell parameters from 9840 reflections

$\theta=3.2-26.4^{\circ}$

$\mu=0.13 \mathrm{~mm}^{-1}$

$T=150 \mathrm{~K}$

Block, orange

$0.34 \times 0.32 \times 0.27 \mathrm{~mm}$

Data collection

Bruker APEXII CCD

$R_{\text {int }}=0.017$

diffractometer

$\varphi$ and $\omega$ scans

34832 measured reflections

$\theta_{\max }=26.4^{\circ}, \theta_{\min }=3.2^{\circ}$

$h=-30 \rightarrow 30$

2944 independent reflections

$k=-15 \rightarrow 15$

2699 reflections with $I>2 \sigma(I)$

$l=-13 \rightarrow 13$

Refinement

Refinement on $F^{2}$

Least-squares matrix: full

$R\left[F^{2}>2 \sigma\left(F^{2}\right)\right]=0.034$

$w R\left(F^{2}\right)=0.102$

$S=1.04$

2944 reflections

241 parameters

6 restraints

Hydrogen site location: mixed

$\mathrm{H}$ atoms treated by a mixture of independent and constrained refinement

$w=1 /\left[\sigma^{2}\left(F_{\mathrm{o}}^{2}\right)+(0.0586 P)^{2}+2.1077 P\right]$

where $P=\left(F_{\mathrm{o}}{ }^{2}+2 F_{\mathrm{c}}{ }^{2}\right) / 3$

$(\Delta / \sigma)_{\max }<0.001$

$\Delta \rho_{\max }=0.29 \mathrm{e} \AA^{-3}$

$\Delta \rho_{\min }=-0.21$ e $\AA^{-3}$ 


\section{Special details}

Geometry. All esds (except the esd in the dihedral angle between two 1.s. planes) are estimated using the full covariance matrix. The cell esds are taken into account individually in the estimation of esds in distances, angles and torsion angles; correlations between esds in cell parameters are only used when they are defined by crystal symmetry. An approximate (isotropic) treatment of cell esds is used for estimating esds involving l.s. planes.

Fractional atomic coordinates and isotropic or equivalent isotropic displacement parameters $\left(\AA^{2}\right)$

\begin{tabular}{|c|c|c|c|c|c|}
\hline & $x$ & $y$ & $z$ & $U_{\text {iso }} * / U_{\text {eq }}$ & Occ. $(<1)$ \\
\hline $\mathrm{C} 1$ & $0.37057(5)$ & $0.39423(9)$ & $0.82909(12)$ & $0.0190(2)$ & \\
\hline $\mathrm{C} 2$ & $0.37833(5)$ & $0.39902(9)$ & $0.97648(12)$ & $0.0207(2)$ & \\
\hline $\mathrm{C} 3$ & $0.48693(5)$ & $0.37742(9)$ & $1.04270(12)$ & $0.0208(2)$ & \\
\hline $\mathrm{C} 4$ & $0.42279(5)$ & $0.38365(8)$ & $0.79361(12)$ & $0.0186(2)$ & \\
\hline $\mathrm{C} 5$ & $0.24165(5)$ & $0.38344(8)$ & $0.50833(12)$ & $0.0192(2)$ & \\
\hline C6 & $0.23130(5)$ & $0.37794(9)$ & $0.36716(12)$ & $0.0204(2)$ & \\
\hline H6 & 0.263906 & 0.380695 & 0.338172 & $0.025^{*}$ & \\
\hline $\mathrm{C} 7$ & $0.17254(5)$ & $0.36834(9)$ & $0.26874(11)$ & $0.0197(2)$ & \\
\hline $\mathrm{C} 8$ & $0.12459(5)$ & $0.36539(9)$ & $0.31079(12)$ & $0.0201(2)$ & \\
\hline H8 & 0.084612 & 0.358679 & 0.243355 & $0.024^{*}$ & \\
\hline C9 & $0.13569(5)$ & $0.37237(9)$ & $0.45277(12)$ & $0.0200(2)$ & \\
\hline $\mathrm{C} 10$ & $0.19423(5)$ & $0.38111(9)$ & $0.55282(12)$ & $0.0203(2)$ & \\
\hline $\mathrm{H} 10$ & 0.201704 & 0.385399 & 0.649417 & $0.024 *$ & \\
\hline C11 & $0.15859(5)$ & $0.35937(9)$ & $0.11562(12)$ & $0.0211(2)$ & \\
\hline $\mathrm{C} 12$ & $0.19700(5)$ & $0.35182(11)$ & $-0.05977(12)$ & $0.0264(3)$ & \\
\hline $\mathrm{H} 12 \mathrm{~A}$ & 0.177183 & 0.284238 & -0.096410 & $0.040^{*}$ & \\
\hline H12B & 0.235389 & 0.355077 & -0.070599 & $0.040^{*}$ & \\
\hline $\mathrm{H} 12 \mathrm{C}$ & 0.171209 & 0.410245 & -0.111573 & $0.040^{*}$ & \\
\hline $\mathrm{C} 13$ & $0.08236(5)$ & $0.37078(10)$ & $0.49220(12)$ & $0.0231(3)$ & \\
\hline C14 & $0.04671(6)$ & $0.39831(12)$ & $0.66992(14)$ & $0.0307(3)$ & \\
\hline H14A & 0.022390 & 0.461429 & 0.630597 & $0.046^{*}$ & \\
\hline H14B & 0.062181 & 0.401216 & 0.772194 & $0.046^{*}$ & \\
\hline $\mathrm{H} 14 \mathrm{C}$ & 0.021864 & 0.334851 & 0.636695 & $0.046^{*}$ & \\
\hline N1 & $0.43716(4)$ & $0.38898(8)$ & $1.07229(10)$ & $0.0215(2)$ & \\
\hline $\mathrm{H} 1 \mathrm{~N}$ & 0.445330 & 0.388946 & 1.160377 & $0.026(4)^{*}$ & \\
\hline $\mathrm{N} 2$ & $0.47752(4)$ & $0.37483(8)$ & $0.90340(10)$ & $0.0209(2)$ & \\
\hline $\mathrm{H} 2 \mathrm{~N}$ & 0.510968 & 0.367522 & 0.886519 & $0.032(4)^{*}$ & \\
\hline N3 & $0.31416(4)$ & $0.39456(7)$ & $0.73771(10)$ & $0.0197(2)$ & \\
\hline N4 & $0.30173(4)$ & $0.38952(8)$ & $0.60489(10)$ & $0.0202(2)$ & \\
\hline $\mathrm{H} 4 \mathrm{~N}$ & 0.329280 & 0.391432 & 0.572748 & $0.041(5)^{*}$ & \\
\hline $\mathrm{O} 1$ & $0.33809(4)$ & $0.41106(8)$ & $1.01707(9)$ & $0.0285(2)$ & \\
\hline $\mathrm{O} 2$ & $0.53768(4)$ & $0.37191(8)$ & $1.13392(9)$ & $0.0279(2)$ & \\
\hline $\mathrm{O} 3$ & $0.41946(4)$ & $0.38163(7)$ & $0.67172(8)$ & $0.0229(2)$ & \\
\hline $\mathrm{O} 4$ & $0.10827(4)$ & $0.35077(8)$ & $0.02776(9)$ & $0.0310(2)$ & \\
\hline O5 & $0.20773(4)$ & $0.36060(7)$ & $0.08791(8)$ & $0.0250(2)$ & \\
\hline O6 & $0.03258(4)$ & $0.35068(10)$ & $0.41153(10)$ & $0.0431(3)$ & \\
\hline $\mathrm{O} 7$ & $0.09668(4)$ & $0.39450(8)$ & $0.62583(9)$ & $0.0292(2)$ & \\
\hline OW1 & $0.3463(2)$ & $0.3484(4)$ & $0.3151(6)$ & $0.0466(19)$ & $0.224(5)$ \\
\hline
\end{tabular}


Atomic displacement parameters $\left(\AA^{2}\right)$

\begin{tabular}{lllllll}
\hline & $U^{11}$ & $U^{22}$ & $U^{33}$ & $U^{12}$ & $U^{13}$ & $U^{23}$ \\
\hline C1 & $0.0158(5)$ & $0.0212(5)$ & $0.0187(5)$ & $-0.0008(4)$ & $0.0054(4)$ & $-0.0001(4)$ \\
C2 & $0.0184(5)$ & $0.0233(6)$ & $0.0203(6)$ & $-0.0022(4)$ & $0.0074(5)$ & $-0.0026(4)$ \\
C3 & $0.0192(5)$ & $0.0237(6)$ & $0.0179(5)$ & $0.0009(4)$ & $0.0054(4)$ & $-0.0003(4)$ \\
C4 & $0.0166(5)$ & $0.0198(5)$ & $0.0175(5)$ & $-0.0002(4)$ & $0.0047(4)$ & $0.0010(4)$ \\
C5 & $0.0157(5)$ & $0.0195(5)$ & $0.0193(5)$ & $-0.0003(4)$ & $0.0034(4)$ & $0.0016(4)$ \\
C6 & $0.0188(5)$ & $0.0220(5)$ & $0.0209(6)$ & $0.0006(4)$ & $0.0081(4)$ & $0.0018(4)$ \\
C7 & $0.0203(5)$ & $0.0198(5)$ & $0.0177(6)$ & $0.0005(4)$ & $0.0061(4)$ & $0.0010(4)$ \\
C8 & $0.0171(5)$ & $0.0211(5)$ & $0.0189(5)$ & $-0.0002(4)$ & $0.0034(4)$ & $0.0003(4)$ \\
C9 & $0.0173(5)$ & $0.0223(5)$ & $0.0193(5)$ & $-0.0007(4)$ & $0.0061(4)$ & $0.0006(4)$ \\
C10 & $0.0197(5)$ & $0.0229(5)$ & $0.0172(5)$ & $-0.0006(4)$ & $0.0061(4)$ & $0.0006(4)$ \\
C11 & $0.0210(5)$ & $0.0222(5)$ & $0.0192(5)$ & $0.0008(4)$ & $0.0070(4)$ & $0.0011(4)$ \\
C12 & $0.0253(6)$ & $0.0365(7)$ & $0.0180(6)$ & $0.0003(5)$ & $0.0092(5)$ & $0.0008(5)$ \\
C13 & $0.0185(6)$ & $0.0295(6)$ & $0.0192(5)$ & $-0.0006(4)$ & $0.0053(4)$ & $0.0002(4)$ \\
C14 & $0.0221(6)$ & $0.0462(8)$ & $0.0262(6)$ & $-0.0023(5)$ & $0.0121(5)$ & $-0.0036(5)$ \\
N1 & $0.0184(5)$ & $0.0310(5)$ & $0.0141(5)$ & $-0.0001(4)$ & $0.0054(4)$ & $-0.0023(4)$ \\
N2 & $0.0149(5)$ & $0.0304(5)$ & $0.0167(5)$ & $0.0025(4)$ & $0.0056(4)$ & $0.0005(4)$ \\
N3 & $0.0174(5)$ & $0.0219(5)$ & $0.0181(5)$ & $-0.0007(3)$ & $0.0053(4)$ & $-0.0001(3)$ \\
N4 & $0.0153(5)$ & $0.0270(5)$ & $0.0176(5)$ & $-0.0005(3)$ & $0.0055(4)$ & $0.0017(4)$ \\
O1 & $0.0192(4)$ & $0.0439(5)$ & $0.0244(4)$ & $-0.0026(4)$ & $0.0108(3)$ & $-0.0066(4)$ \\
O2 & $0.0190(4)$ & $0.0441(5)$ & $0.0169(4)$ & $0.0043(4)$ & $0.0032(3)$ & $-0.0004(3)$ \\
O3 & $0.0178(4)$ & $0.0344(5)$ & $0.0161(4)$ & $-0.0001(3)$ & $0.0062(3)$ & $0.0014(3)$ \\
O4 & $0.0211(4)$ & $0.0510(6)$ & $0.0187(4)$ & $-0.0024(4)$ & $0.0056(3)$ & $-0.0028(4)$ \\
O5 & $0.0204(4)$ & $0.0370(5)$ & $0.0172(4)$ & $0.0008(3)$ & $0.0071(3)$ & $0.0015(3)$ \\
O6 & $0.0175(4)$ & $0.0853(8)$ & $0.0252(5)$ & $-0.0085(5)$ & $0.0069(4)$ & $-0.0130(5)$ \\
O7 & $0.0190(4)$ & $0.0498(6)$ & $0.0193(4)$ & $-0.0038(4)$ & $0.0080(3)$ & $-0.0038(4)$ \\
OW1 & $0.031(3)$ & $0.056(3)$ & $0.058(3)$ & $0.001(2)$ & $0.024(2)$ & $0.004(2)$ \\
& & & & & & \\
\hline
\end{tabular}

Geometric parameters $\left(A,{ }^{\circ}\right)$

\begin{tabular}{llll}
\hline $\mathrm{C} 1-\mathrm{N} 3$ & $1.3223(15)$ & $\mathrm{C} 9-\mathrm{C} 10$ & $1.3945(16)$ \\
$\mathrm{C} 1-\mathrm{C} 4$ & $1.4557(16)$ & $\mathrm{C} 9-\mathrm{C} 13$ & $1.5006(16)$ \\
$\mathrm{C} 1-\mathrm{C} 2$ & $1.4708(15)$ & $\mathrm{C} 10-\mathrm{H} 10$ & 0.9500 \\
$\mathrm{C} 2-\mathrm{O} 1$ & $1.2140(14)$ & $\mathrm{C} 11-\mathrm{O} 4$ & $1.2068(14)$ \\
$\mathrm{C} 2-\mathrm{N} 1$ & $1.3864(14)$ & $\mathrm{C} 11-\mathrm{O} 5$ & $1.3298(14)$ \\
$\mathrm{C} 3-\mathrm{O} 2$ & $1.2242(14)$ & $\mathrm{C} 12-\mathrm{O} 5$ & $1.4581(14)$ \\
$\mathrm{C} 3-\mathrm{N} 1$ & $1.3638(15)$ & $\mathrm{C} 12-\mathrm{H} 12 \mathrm{~A}$ & 0.9800 \\
$\mathrm{C} 3-\mathrm{N} 2$ & $1.3759(15)$ & $\mathrm{C} 12-\mathrm{H} 12 \mathrm{~B}$ & 0.9800 \\
$\mathrm{C} 4-\mathrm{O} 3$ & $1.2387(14)$ & $\mathrm{C} 12-\mathrm{H} 12 \mathrm{C}$ & 0.9800 \\
$\mathrm{C} 4-\mathrm{N} 2$ & $1.3724(14)$ & $\mathrm{C} 13-\mathrm{O} 6$ & $1.1944(15)$ \\
$\mathrm{C} 5-\mathrm{C} 6$ & $1.3909(16)$ & $\mathrm{C} 13-\mathrm{O} 7$ & $1.3280(15)$ \\
$\mathrm{C} 5-\mathrm{C} 10$ & $1.3968(16)$ & $\mathrm{C} 14-\mathrm{O} 7$ & 0.9800 \\
$\mathrm{C} 5-\mathrm{N} 4$ & $1.4080(14)$ & $\mathrm{C} 14-\mathrm{H} 14 \mathrm{~A}$ & 0.9800 \\
$\mathrm{C} 6-\mathrm{C} 7$ & $1.3936(16)$ & $\mathrm{C} 14-\mathrm{H} 14 \mathrm{~B}$ & 0.9800 \\
$\mathrm{C} 6-\mathrm{H} 6$ & 0.9500 & $\mathrm{C} 14-\mathrm{H} 14 \mathrm{C}$ & 0.8580 \\
$\mathrm{C} 7-\mathrm{C} 8$ & $1.3926(16)$ & $\mathrm{N} 1-\mathrm{H} 1 \mathrm{~N}$ &
\end{tabular}




\begin{tabular}{|c|c|c|c|}
\hline $\mathrm{C} 7-\mathrm{C} 11$ & $1.4970(15)$ & $\mathrm{N} 2-\mathrm{H} 2 \mathrm{~N}$ & 0.8982 \\
\hline $\mathrm{C} 8-\mathrm{C} 9$ & $1.3963(16)$ & $\mathrm{N} 3-\mathrm{N} 4$ & $1.2950(14)$ \\
\hline $\mathrm{C} 8-\mathrm{H} 8$ & 0.9500 & $\mathrm{~N} 4-\mathrm{H} 4 \mathrm{~N}$ & 0.8557 \\
\hline $\mathrm{N} 3-\mathrm{C} 1-\mathrm{C} 4$ & $124.93(10)$ & $\mathrm{O} 4-\mathrm{C} 11-\mathrm{O} 5$ & $124.04(10)$ \\
\hline $\mathrm{N} 3-\mathrm{C} 1-\mathrm{C} 2$ & $114.97(10)$ & $\mathrm{O} 4-\mathrm{C} 11-\mathrm{C} 7$ & $123.45(10)$ \\
\hline $\mathrm{C} 4-\mathrm{C} 1-\mathrm{C} 2$ & $120.00(10)$ & $\mathrm{O} 5-\mathrm{C} 11-\mathrm{C} 7$ & $112.50(9)$ \\
\hline $\mathrm{O} 1-\mathrm{C} 2-\mathrm{N} 1$ & $119.97(10)$ & $\mathrm{O} 5-\mathrm{C} 12-\mathrm{H} 12 \mathrm{~A}$ & 109.5 \\
\hline $\mathrm{O} 1-\mathrm{C} 2-\mathrm{C} 1$ & $125.19(10)$ & $\mathrm{O} 5-\mathrm{C} 12-\mathrm{H} 12 \mathrm{~B}$ & 109.5 \\
\hline $\mathrm{N} 1-\mathrm{C} 2-\mathrm{C} 1$ & $114.84(9)$ & $\mathrm{H} 12 \mathrm{~A}-\mathrm{C} 12-\mathrm{H} 12 \mathrm{~B}$ & 109.5 \\
\hline $\mathrm{O} 2-\mathrm{C} 3-\mathrm{N} 1$ & $122.53(10)$ & $\mathrm{O} 5-\mathrm{C} 12-\mathrm{H} 12 \mathrm{C}$ & 109.5 \\
\hline $\mathrm{O} 2-\mathrm{C} 3-\mathrm{N} 2$ & $121.04(10)$ & $\mathrm{H} 12 \mathrm{~A}-\mathrm{C} 12-\mathrm{H} 12 \mathrm{C}$ & 109.5 \\
\hline $\mathrm{N} 1-\mathrm{C} 3-\mathrm{N} 2$ & $116.41(10)$ & $\mathrm{H} 12 \mathrm{~B}-\mathrm{C} 12-\mathrm{H} 12 \mathrm{C}$ & 109.5 \\
\hline $\mathrm{O} 3-\mathrm{C} 4-\mathrm{N} 2$ & $120.22(10)$ & $\mathrm{O} 6-\mathrm{C} 13-\mathrm{O} 7$ & $124.04(11)$ \\
\hline $\mathrm{O} 3-\mathrm{C} 4-\mathrm{C} 1$ & $123.21(10)$ & $\mathrm{O} 6-\mathrm{C} 13-\mathrm{C} 9$ & $123.36(11)$ \\
\hline $\mathrm{N} 2-\mathrm{C} 4-\mathrm{C} 1$ & $116.56(10)$ & $\mathrm{O} 7-\mathrm{C} 13-\mathrm{C} 9$ & $112.60(9)$ \\
\hline $\mathrm{C} 6-\mathrm{C} 5-\mathrm{C} 10$ & $121.20(10)$ & $\mathrm{O} 7-\mathrm{C} 14-\mathrm{H} 14 \mathrm{~A}$ & 109.5 \\
\hline $\mathrm{C} 6-\mathrm{C} 5-\mathrm{N} 4$ & $117.59(10)$ & $\mathrm{O} 7-\mathrm{C} 14-\mathrm{H} 14 \mathrm{~B}$ & 109.5 \\
\hline $\mathrm{C} 10-\mathrm{C} 5-\mathrm{N} 4$ & $121.20(10)$ & $\mathrm{H} 14 \mathrm{~A}-\mathrm{C} 14-\mathrm{H} 14 \mathrm{~B}$ & 109.5 \\
\hline $\mathrm{C} 5-\mathrm{C} 6-\mathrm{C} 7$ & $119.25(10)$ & $\mathrm{O} 7-\mathrm{C} 14-\mathrm{H} 14 \mathrm{C}$ & 109.5 \\
\hline $\mathrm{C} 5-\mathrm{C} 6-\mathrm{H} 6$ & 120.4 & $\mathrm{H} 14 \mathrm{~A}-\mathrm{C} 14-\mathrm{H} 14 \mathrm{C}$ & 109.5 \\
\hline $\mathrm{C} 7-\mathrm{C} 6-\mathrm{H} 6$ & 120.4 & $\mathrm{H} 14 \mathrm{~B}-\mathrm{C} 14-\mathrm{H} 14 \mathrm{C}$ & 109.5 \\
\hline $\mathrm{C} 8-\mathrm{C} 7-\mathrm{C} 6$ & $120.52(10)$ & $\mathrm{C} 3-\mathrm{N} 1-\mathrm{C} 2$ & $126.63(9)$ \\
\hline $\mathrm{C} 8-\mathrm{C} 7-\mathrm{C} 11$ & $117.69(10)$ & $\mathrm{C} 3-\mathrm{N} 1-\mathrm{H} 1 \mathrm{~N}$ & 112.8 \\
\hline $\mathrm{C} 6-\mathrm{C} 7-\mathrm{C} 11$ & $121.79(10)$ & $\mathrm{C} 2-\mathrm{N} 1-\mathrm{H} 1 \mathrm{~N}$ & 120.6 \\
\hline $\mathrm{C} 7-\mathrm{C} 8-\mathrm{C} 9$ & $119.52(10)$ & $\mathrm{C} 4-\mathrm{N} 2-\mathrm{C} 3$ & $125.51(10)$ \\
\hline $\mathrm{C} 7-\mathrm{C} 8-\mathrm{H} 8$ & 120.2 & $\mathrm{C} 4-\mathrm{N} 2-\mathrm{H} 2 \mathrm{~N}$ & 119.7 \\
\hline $\mathrm{C} 9-\mathrm{C} 8-\mathrm{H} 8$ & 120.2 & $\mathrm{C} 3-\mathrm{N} 2-\mathrm{H} 2 \mathrm{~N}$ & 114.8 \\
\hline $\mathrm{C} 10-\mathrm{C} 9-\mathrm{C} 8$ & $120.75(11)$ & $\mathrm{N} 4-\mathrm{N} 3-\mathrm{C} 1$ & $120.55(10)$ \\
\hline $\mathrm{C} 10-\mathrm{C} 9-\mathrm{C} 13$ & $121.89(10)$ & $\mathrm{N} 3-\mathrm{N} 4-\mathrm{C} 5$ & $120.38(9)$ \\
\hline $\mathrm{C} 8-\mathrm{C} 9-\mathrm{C} 13$ & $117.35(10)$ & $\mathrm{N} 3-\mathrm{N} 4-\mathrm{H} 4 \mathrm{~N}$ & 121.7 \\
\hline $\mathrm{C} 9-\mathrm{C} 10-\mathrm{C} 5$ & $118.75(10)$ & $\mathrm{C} 5-\mathrm{N} 4-\mathrm{H} 4 \mathrm{~N}$ & 117.9 \\
\hline $\mathrm{C} 9-\mathrm{C} 10-\mathrm{H} 10$ & 120.6 & $\mathrm{C} 11-\mathrm{O} 5-\mathrm{C} 12$ & $115.04(9)$ \\
\hline $\mathrm{C} 5-\mathrm{C} 10-\mathrm{H} 10$ & 120.6 & $\mathrm{C} 13-\mathrm{O} 7-\mathrm{C} 14$ & $115.50(9)$ \\
\hline $\mathrm{N} 3-\mathrm{C} 1-\mathrm{C} 2-\mathrm{O} 1$ & $-5.85(17)$ & $\mathrm{C} 6-\mathrm{C} 7-\mathrm{C} 11-\mathrm{O} 5$ & $0.66(15)$ \\
\hline $\mathrm{C} 4-\mathrm{C} 1-\mathrm{C} 2-\mathrm{O} 1$ & $177.58(11)$ & $\mathrm{C} 10-\mathrm{C} 9-\mathrm{C} 13-\mathrm{O} 6$ & $-170.65(13)$ \\
\hline $\mathrm{N} 3-\mathrm{C} 1-\mathrm{C} 2-\mathrm{N} 1$ & $174.41(9)$ & $\mathrm{C} 8-\mathrm{C} 9-\mathrm{C} 13-\mathrm{O} 6$ & $9.75(18)$ \\
\hline $\mathrm{C} 4-\mathrm{C} 1-\mathrm{C} 2-\mathrm{N} 1$ & $-2.17(15)$ & $\mathrm{C} 10-\mathrm{C} 9-\mathrm{C} 13-\mathrm{O} 7$ & $9.60(16)$ \\
\hline $\mathrm{N} 3-\mathrm{C} 1-\mathrm{C} 4-\mathrm{O} 3$ & $5.51(18)$ & $\mathrm{C} 8-\mathrm{C} 9-\mathrm{C} 13-\mathrm{O} 7$ & $-170.00(10)$ \\
\hline $\mathrm{C} 2-\mathrm{C} 1-\mathrm{C} 4-\mathrm{O} 3$ & $-178.27(10)$ & $\mathrm{O} 2-\mathrm{C} 3-\mathrm{N} 1-\mathrm{C} 2$ & $177.97(11)$ \\
\hline $\mathrm{N} 3-\mathrm{C} 1-\mathrm{C} 4-\mathrm{N} 2$ & $-173.94(10)$ & $\mathrm{N} 2-\mathrm{C} 3-\mathrm{N} 1-\mathrm{C} 2$ & $-0.46(17)$ \\
\hline $\mathrm{C} 2-\mathrm{C} 1-\mathrm{C} 4-\mathrm{N} 2$ & $2.27(15)$ & $\mathrm{O} 1-\mathrm{C} 2-\mathrm{N} 1-\mathrm{C} 3$ & $-178.47(11)$ \\
\hline $\mathrm{C} 10-\mathrm{C} 5-\mathrm{C} 6-\mathrm{C} 7$ & $-0.97(16)$ & $\mathrm{C} 1-\mathrm{C} 2-\mathrm{N} 1-\mathrm{C} 3$ & $1.29(17)$ \\
\hline $\mathrm{N} 4-\mathrm{C} 5-\mathrm{C} 6-\mathrm{C} 7$ & $177.91(10)$ & $\mathrm{O} 3-\mathrm{C} 4-\mathrm{N} 2-\mathrm{C} 3$ & $179.07(11)$ \\
\hline $\mathrm{C} 5-\mathrm{C} 6-\mathrm{C} 7-\mathrm{C} 8$ & $0.67(16)$ & $\mathrm{C} 1-\mathrm{C} 4-\mathrm{N} 2-\mathrm{C} 3$ & $-1.46(16)$ \\
\hline $\mathrm{C} 5-\mathrm{C} 6-\mathrm{C} 7-\mathrm{C} 11$ & $-178.47(10)$ & $\mathrm{O} 2-\mathrm{C} 3-\mathrm{N} 2-\mathrm{C} 4$ & $-177.92(11)$ \\
\hline $\mathrm{C} 6-\mathrm{C} 7-\mathrm{C} 8-\mathrm{C} 9$ & $0.14(16)$ & $\mathrm{N} 1-\mathrm{C} 3-\mathrm{N} 2-\mathrm{C} 4$ & $0.53(17)$ \\
\hline
\end{tabular}




\begin{tabular}{llll}
$\mathrm{C} 11-\mathrm{C} 7-\mathrm{C} 8-\mathrm{C} 9$ & $179.32(10)$ & $\mathrm{C} 4-\mathrm{C} 1-\mathrm{N} 3-\mathrm{N} 4$ & $-2.91(17)$ \\
$\mathrm{C} 7-\mathrm{C} 8-\mathrm{C} 9-\mathrm{C} 10$ & $-0.68(16)$ & $\mathrm{C} 2-\mathrm{C} 1-\mathrm{N} 3-\mathrm{N} 4$ & $-179.30(9)$ \\
$\mathrm{C} 7-\mathrm{C} 8-\mathrm{C} 9-\mathrm{C} 13$ & $178.92(10)$ & $\mathrm{C} 1-\mathrm{N} 3-\mathrm{N} 4-\mathrm{C} 5$ & $176.15(10)$ \\
$\mathrm{C} 8-\mathrm{C} 9-\mathrm{C} 10-\mathrm{C} 5$ & $0.40(16)$ & $\mathrm{C} 6-\mathrm{C} 5-\mathrm{N} 4-\mathrm{N} 3$ & $-179.84(10)$ \\
$\mathrm{C} 13-\mathrm{C} 9-\mathrm{C} 10-\mathrm{C} 5$ & $-179.18(10)$ & $\mathrm{C} 10-\mathrm{C} 5-\mathrm{N} 4-\mathrm{N} 3$ & $-0.97(16)$ \\
$\mathrm{C} 6-\mathrm{C} 5-\mathrm{C} 10-\mathrm{C} 9$ & $0.43(16)$ & $\mathrm{O} 4-\mathrm{C} 11-\mathrm{O} 5-\mathrm{C} 12$ & $0.65(16)$ \\
$\mathrm{N} 4-\mathrm{C} 5-\mathrm{C} 10-\mathrm{C} 9$ & $-178.40(10)$ & $\mathrm{C} 7-\mathrm{C} 11-\mathrm{O} 5-\mathrm{C} 12$ & $179.85(9)$ \\
$\mathrm{C} 8-\mathrm{C} 7-\mathrm{C} 11-\mathrm{O} 4$ & $0.70(17)$ & $\mathrm{O} 6-\mathrm{C} 13-\mathrm{O} 7-\mathrm{C} 14$ & $-1.96(19)$ \\
$\mathrm{C} 6-\mathrm{C} 7-\mathrm{C} 11-\mathrm{O} 4$ & $179.87(11)$ & $\mathrm{C} 9-\mathrm{C} 13-\mathrm{O} 7-\mathrm{C} 14$ & $177.79(10)$ \\
$\mathrm{C} 8-\mathrm{C} 7-\mathrm{C} 11-\mathrm{O} 5$ & $-178.51(10)$ & & \\
\hline
\end{tabular}

Hydrogen-bond geometry $\left(\AA,{ }^{\circ}\right)$

$\mathrm{Cg} 2$ is the centroid of the $\mathrm{C} 5-\mathrm{C} 10$ benzene ring.

\begin{tabular}{lllll}
\hline$D-\mathrm{H} \cdots A$ & $D-\mathrm{H}$ & $\mathrm{H} \cdots A$ & $D \cdots A$ & $D-\mathrm{H} \cdots A$ \\
\hline $\mathrm{N} 1-\mathrm{H} 1 N^{\cdots} \mathrm{O} 2^{2}$ & 0.86 & 2.03 & $2.8800(13)$ & 174 \\
$\mathrm{~N} 2-\mathrm{H} 2 N \cdots \mathrm{O} 3^{\text {ii }}$ & 0.90 & 2.01 & $2.8931(15)$ & 168 \\
$\mathrm{~N} 4-\mathrm{H} 4 N \cdots \mathrm{O} 3$ & 0.86 & 2.02 & $2.6571(15)$ & 131 \\
$\mathrm{~N} 4-\mathrm{H} 4 N \cdots \mathrm{O} 1^{\text {iii }}$ & 0.86 & 2.59 & $2.9302(14)$ & 105 \\
$\mathrm{C} 6-\mathrm{H} 6 \cdots \mathrm{O} 1$ & 0.95 & 2.14 & $3.061(6)$ & 163 \\
$\mathrm{C} 12-\mathrm{H} 12 B \cdots \mathrm{O} 1^{\text {iv }}$ & 0.98 & 2.39 & $3.2743(17)$ & 149 \\
$\mathrm{C} 14-\mathrm{H} 14 B \cdots \mathrm{O} 4^{\mathrm{v}}$ & 0.98 & 2.53 & $3.4754(16)$ & 163 \\
$\mathrm{C} 12-\mathrm{H} 12 C \cdots C g 2^{\mathrm{iii}}$ & 0.98 & 2.73 & $3.4717(15)$ & 133
\end{tabular}

Symmetry codes: (i) $-x+1, y,-z+5 / 2$; (ii) $-x+1, y,-z+3 / 2$; (iii) $x,-y+1, z-1 / 2$; (iv) $x, y, z-1$; (v) $x, y, z+1$. 